\title{
LA FAMILIA FAGACEAE EN EL BOSQUE MESÓFILO DE MONTAÑA DE MÉXICO
}

\author{
Susana Valencia-A..$^{1,3}$ y Martha Gual-Díaz ${ }^{2}$ \\ ${ }^{1}$ Herbario de la Facultad de Ciencias, Departamento de Biología Comparada, \\ Universidad Nacional Autónoma de México, México, D.F., México \\ ${ }^{2}$ Comisión Nacional para el Conocimiento y Uso de la Biodiversidad (CONABIO), México, D.F., México \\ ${ }^{3}$ Autor para la correspondencia: querc2002@yahoo.com.mx
}

\begin{abstract}
Resumen: El bosque mesófilo de montaña es uno de los tipos de vegetación más amenazados, donde la familia Fagaceae juega un papel ecológico clave, por lo que su conocimiento en esta comunidad es importante para su conservación. Con base en datos de herbario y de campo, de literatura y del Sistema de Información de los Bosques Mesófilos de Montaña en México, elaborado por la Comisión Nacional para el Conocimiento y Uso de la Biodiversidad, se presenta un análisis de la familia Fagaceae en el bosque mesófilo de montaña, con énfasis en las 13 regiones prioritarias propuestas por la Comisión Nacional para el Conocimiento y Uso de la Biodiversidad para la conservación del bosque mesófilo de montaña. Asimismo, se compiló la categoría de riesgo de las Fagaceae en este bosque. Los resultados muestran que en este ecosistema están presentes 47 especies de Fagaceae, 46 de Quercus (32 de la sección Lobatae y 14 de la sección Quercus) y una de Fagus; de ellas, 13 se consideran exclusivas o casi exclusivas de esta comunidad y endémicas de México. De las 13 regiones prioritarias, la región III (Huasteca Alta Hidalguense) resultó la de mayor diversidad con 26 especies, mientras que la región VII (Los Tuxtlas) es la menos diversa con sólo dos especies. El análisis de diversidad $\beta$ muestra un recambio de medio a alto que va de 0.41 a 1 entre las 13 regiones. Las regiones más similares en cuanto a especies de Fagaceae fueron la III (Huasteca Alta Hidalguense) y la V (Centro de Veracruz). Las especies de Fagaceae señaladas con alguna categoría de riesgo o amenaza son 41. Destacan ocho especies de Lobatae que se consideran críticamente amenazadas y cinco de Quercus en la misma categoría.
\end{abstract}

Palabras clave: conservación, distribución, Fagus, Quercus, regiones prioritarias, riqueza de especies.

\begin{abstract}
Cloud forest in Mexico is one of the most fragile biomes, wherein some species of Fagaceae family play a key ecological role. Based on data from reviews of herbarium material, publications of Mexican cloud forest, field visits and Mexico's cloud forest biodiversity information system housed by Comisión Nacional para el Conocimiento y Uso de la Biodiversidad, we present an analysis of the Fagaceae family in the cloud forests of Mexico, with special emphasis in the 13 main regions proposed by Comisión Nacional para el Conocimiento y Uso de la Biodiversidad as priority for the conservation of cloud forests in the country. Likewise, we compiled the risk categories of the Fagaceae family from the cloud forest. Results show that the family is represented in this community by 46 species of Quercus (32 from the Lobatae section and 14 from the Quercus section), and one species of Fagus. Thirteen of these species are considered exclusive or almost exclusive to this community and endemic to Mexico. Among the priority regions, region III (Huasteca Alta Hidalguense) is the most diverse with 26 species; while region VII (Los Tuxtlas) is the less diverse with only two species. The $\beta$ diversity analysis shows intermediate to high rates between the 13 regions (from 0.41-1). Most similar regions in Fagaceae species were III (Huasteca Alta Hidalguense) and V (Centro de Veracruz). The Fagaceae species of Mexican cloud forest classified in some level of risk or threat are 41. Among them, we highlight eight oak species of section Lobatae, considered critically endangered, and five of section Quercus in the same category.
\end{abstract}

Keywords: conservation, distribution, Fagus, Quercus, priority regions, species richness.

$\mathbf{E}$ 1 bosque mesófilo de montaña (BMM) incluye un conjunto de comunidades heterogéneas que difieren en cuanto a la fisiografía en la que se desarrollan, en su estructura y composición florística. Entre las principales características que permiten distinguir a este bosque se encuentran la mezcla de elementos de afinidad boreal y tropical (González-
Espinosa et al., 2012); su compleja estructura horizontal y vertical, dada por la diversidad de hábitos de crecimiento y; la abundancia de epífitas, pteridofitas y trepadoras leñosas, todo ello enmarcado por condiciones ambientales mésicas con alta precipitación pluvial y humedad atmosférica (Rzedowski, 1978). Otro atributo del BMM es la alta riqueza 
específica por unidad de área (Rzedowski, 1996), considerada ocho veces mayor a la observada en los bosques de coníferas y de encinos (Valdez et al., 2003).

El BMM se distribuye naturalmente de forma fragmentada en las regiones montanas y húmedas de México. Diferentes estimaciones indican que ocupa entre el 0.5 y $7 \%$ del territorio nacional (Flores et al., 1971; Rzedowski, 1978, 1996; Chaverri-Polini, 1998; Cavazos-Camacho y Treviño-Garza, 2000; Cruz-Cárdenas et al., 2012). Presenta su límite boreal en la región noreste del país, en el estado de Nuevo León. En el oeste de México se localiza desde Sinaloa hasta Chiapas, así como en las regiones más húmedas de la Faja Volcánica Transmexicana (Valdez et al., 2003; Cruz-Cárdenas et al., 2012). La distribución más oriental de estos bosques la reporta Almeida-Cerino (2008), y corresponde a una pequeña área localizada en el municipio de Huimanguillo, en el estado de Tabasco, confirmada por la M. en C. Ofelia Castillo Acosta (com. pers., 2012) de la Universidad Juárez Autónoma de Tabasco. Puede desarrollarse a una altitud que va de los 1,000 a los 3,000 m (Villaseñor, 2010), aunque es más común entre los 1,500 y 2,500 m (González-Espinosa et al., 2011).

Las especies de la familia Fagaceae, particularmente los géneros Quercus y Fagus son de los elementos boreales más importantes y distintivos del BMM (Téllez-Valdés et al., 2006; González-Espinosa et al., 2012; Olvera-Vargas y Figueroa-Rangel, 2012). En México, el género Fagus, comúnmente conocido como "haya", tiene una sola especie, Fagus grandifolia subsp. mexicana, con una distribución fragmentada y limitada a las regiones montañosas templadas y húmedas del noreste del país, mientras que Quercus tiene una amplia distribución, sus especies se conocen como "encinos" o "robles", y cuenta con más de 160 especies mexicanas (Valencia-A., 2004).

Diversos estudios estructurales del BMM muestran a los "encinos" o "robles" como elementos con un alto valor de importancia (Meave et al., 1992; Luna et al., 2007; MejíaDomínguez et al., 2004; Olvera-Vargas y Figueroa-Rangel, 2012). Recientemente, Juárez-Medina (2013) analizó la diversidad de tres BMM ubicados en tres regiones diferentes de México y encontró que los bosques, en donde los encinos son los elementos dominantes, tienen más diversidad que aquellos dominados por otros géneros. Tanto los encinos como las hayas, juegan un papel fundamental en los procesos ecosistémicos al albergar una diversidad alta de especies de plantas epífitas, animales, hongos y bacterias; además, proporcionan alimento a un gran número de organismos y son importantes en la retención y el balance del agua en los bosques, por lo que se pueden considerar como grupos fundadores o clave en los ecosistemas en los que se presentan (Ellison et al., 2005; González-Espinosa et al., 2012). Por lo tanto, su pérdida o remoción tendría consecuencias desfavorables o incluso catastróficas en la biota asociada, en la funcionalidad del ecosistema y en la estabilidad del mismo.

De acuerdo con Challenger (1998) y CONABIO (2010), el BMM es el ecosistema más amenazado, por lo que el conocimiento de los elementos que lo conforman es esencial para su conservación. Si los encinos y las hayas son elementos clave en estas comunidades, resulta imperativo conocer qué especies de Fagaceae crecen en el BMM, cómo se distribuyen éstas en las diferentes regiones que ocupa este bosque en México y cuál es su estatus de riesgo.

Algunos estudios del BMM incluyen datos en torno a la riqueza de la familia Fagaceae, como el de Luna-Vega y Magallón (2010), el de Villaseñor (2010) y el de González-Espinosa et al. (2011). Sin embargo, estos trabajos son disímiles en la cifra de especies de Fagaceae que citan para el BMM, tampoco la tratan de forma única o prioritaria, ni analizan a la familia Fagaceae, y no incluyen a todas las especies de la misma que forman parte de este bosque, o incluyen especies que no forman parte de este tipo de vegetación, lo cual puede deberse a errores en la identificación de las especies (por su elevada variación y complejidad taxonómica) o debido a cambios taxonómicos o nomenclaturales. Por lo que hay varias preguntas aún sin responder, como son: ¿cuántas y cuáles especies de Fagaceae forman parte del BMM de México? ¿Cómo se distribuyen estas especies en las diferentes regiones en donde ocurre este bosque? ¿En dónde se concentra la mayor riqueza y endemismo de especies de Fagaceae del BMM? ¿Todas las especies de Fagaceae del BMM requieren del mismo nivel de atención para su estudio y conservación? El objetivo de este trabajo fue responder a estas preguntas por medio del estudio y análisis de la riqueza específica de la familia Fagaceae en el BMM; de su distribución general y, en particular, en las regiones prioritarias propuestas por la CONABIO (2010), del análisis de similitud y de diversidad $\beta$ entre las mismas regiones (utilizando a las especies de la familia); y de la compilación del nivel de riesgo que tienen las especies de Fagaceae del BMM. Se pretende que los datos de esta investigación contribuyan en la toma de decisiones para priorizar estudios futuros en las especies de Fagaceae del BMM, lo cual a su vez repercutirá en la conservación de este tipo de vegetación.

\section{Materiales y métodos}

Para conocer la riqueza de especies de Fagaceae del BMM se analizó la información obtenida de la literatura con respecto a listas florísticas publicadas para este bosque y del Sistema de Información de los Bosques Mesófilos de Montaña en México (SI-BMM) proporcionada por CONABIO, con ello se obtuvo una lista preliminar de las especies de la familia Fagaceae para este ecosistema. La lista fue depurada, en algunos casos corroborando la identificación correcta de los especímenes en herbarios (FCME y MEXU), o confirmando su presencia en dicho bosque y en las zonas referidas en la literatura, así como con datos de campo y de herbario acumulados a lo largo de 25 años de trabajo con la familia Fagaceae en México. 
Las especies o nombres que se presentan a continuación fueron eliminados de la lista preliminar, entre paréntesis se escribe la fuente en la que fueron citados para el BMM: Quercus acutifolia (Alcántara y Luna, 2001; Ruíz-Jiménez et al., 2012); Q. aristata (Luna et al., 1988); Q. canbyi (Valdez et al., 2003; Villaseñor, 2010); Q. clivicola (Briones, 1991); Q. coccolobifolia (Valdez et al., 2003); Q. conspersa (Aguilar et al., 2001); Q. convallata (Johnston et al., 1989); Q. desertícola (Ponce-Vargas et al., 2006); Q. diversifolia y Q. eduardii (Mayorga et al., 1998); Q. eugeniifolia (Villaseñor, 2010; Ruíz-Jiménez et al., 2012); Q. gentryi (Medina et al., 2000); Q. glaucescens (Aguilar et al., 2001); Q. glaucoides (Martínez et al., 2007); Q. grahamii (Constance y Breedlove, 1994); Q. greggii (Vargas-Ajuria, 1982; Valdez et al., 2003); Q. hintonii (Orozco-Villa, 1995); Q. floccosa (Luna et al., 2007); Q. leiophylla (Alcántara y Luna, 1997); Q. liebmanii (Fonseca et al., 2001); Q. magnoliifolia (Luna et al., 1999); Q. microphylla (Vargas-Ajuria, 1982); Q. muehlenbergii (Valdez et al., 2003); Q. ocoteifolia (MejíaDomínguez et al., 2004; Lorea-Hernández y Munn-Estrada, 2005; González-Espinosa et al., 2011; Ruíz-Jiménez et al., 2012); Q. oleoides (Aguilar et al., 2001); Q. opaca (Johnston et al., 1989); Q. planipocula (Aguilar et al., 2001); Q. praineana (Cuevas-Guzmán, 1994); Q. repanda (VargasAjuria, 1982); Q. salicifolia (Constance y Breedlove, 1994; Villaseñor, 2010); Q. splendens (Campos-Villanueva y Villaseñor, 1995; Ruíz-Jiménez et al., 2012); Q. subspathulata y Q. urbanii (Martínez et al., 2007). Su eliminación atendió a que no son integrantes del BMM, sino características del bosque tropical caducifolio, del bosque de Quercus, del bosque de coníferas y Quercus, como es el caso de Q. glaucoides, Q. magnoliifolia, $Q$. liebmani, $Q$. eduardii, $Q$. acutifolia, $Q$. splendens y $Q$. urbanii, o incluso crecen en el bosque tropical subcaducifolio, como $Q$. oleoides y $Q$. glaucescens.

Algunas de las especies del párrafo anterior fueron equívocamente incluidas por diversos autores como elementos del BMM debido a problemas taxonómicos o errores en la identificación de las colectas. Por ejemplo, Q. acutifolia y Q. grahamii se identificaron erróneamente como tales para los BMM de Hidalgo, San Luis Potosí o Veracruz, pero estas especies están ausentes en dichos estados y a tales colectas les corresponde el nombre de $Q$. sartorii, que es muy abundante en los estados señalados, o bien se trata de $Q$. xalapensis de distribución más limitada; estas cuatro especies es fácil confundirlas. En situación similar está la recientemente descrita $Q$. delgadoana (Valencia et al., 2011), que originalmente fue mal identificada como $Q$. eugeniifolia, que no está presente en México. Otro caso es el de $Q$. nixoniana, que se identificó con desacierto como Q. salicifolia, esta última especie con una distribución restringida al bosque tropical subcaducifolio de la costa del Pacífico.

También se excluyeron nombres que no son aceptados y que actualmente se consideran sinónimos de otros que sí fueron incluidos, como Quercus leiophylla, cuyo nom- bre aceptado es $Q$. lancifolia, y $Q$. ocoteifolia sinónimo de $Q$. laurina. Asimismo, fue excluida $Q$. oocarpa porque sus límites morfológicos con $Q$. insignis son inciertos; las muestras previamente identificadas como $Q$. oocarpa de los estados de Guerrero, Jalisco y Nayarit, en este trabajo fueron tratadas como $Q$. insignis. Tampoco se incluyó $Q$. vicentensis porque sus límites morfológicos no son nítidos con $Q$. martinezii.

Por otro lado, sí se incluyeron en la lista: Quercus castanea, $Q$. crassifolia, $Q$. duratifolia, $Q$. furfuracea, $Q$. iltisii, Q. laeta, Q. laurina, Q. mexicana, Q. obtusata, Q. peduncularis, $Q$. scytophylla y $Q$. ariifolia, que son características de comunidades como el bosque de coníferas y de Quercus, y bosque de Quercus, pero eventualmente también forman parte de los BMM menos húmedos y más fríos. Quercus ariifolia es considerada por las autoras como un taxon diferente a $Q$. rugosa, por lo que ambas fueron incluidas en este análisis.

Después de ser depurada, la lista de la familia Fagaceae fue analizada, resaltando las especies que se consideran exclusivas (sólo se presentan en el BMM) o casi exclusivas del BMM (rara vez se les encuentra creciendo en otros tipos de vegetación) y las especies endémicas de México. Posteriormente, y tomando como referencia la propuesta de las regiones prioritarias para la conservación del BMM en México, de la CONABIO (2010; Figura 1), se analizó la distribución de las especies en tales regiones. Es necesario aclarar que, aunque los polígonos de estas regiones se muestran gráficamente con una distribución continua y amplia del BMM, este tipo de vegetación no está presente en la totalidad del

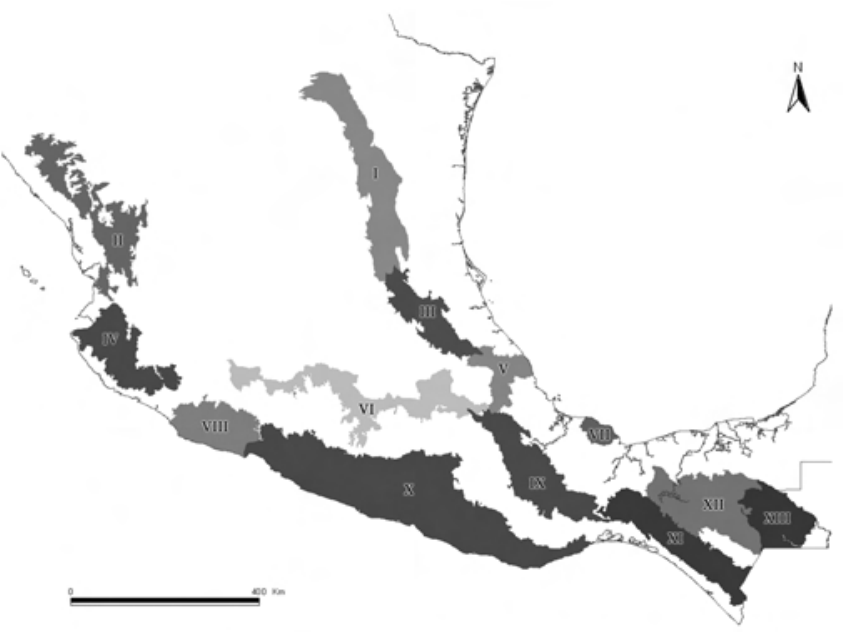

Figura 1. Regiones prioritas de conservación del BMM en México. I. Sierra Madre Oriental Plegada; II. Serranías de Nayarit; III. Huasteca alta Hidalguense; IV Sierra Madre del Sur y Franja Neovolcánica de Jalisco; V. Centro de Veracruz; VI. Cuenca alta del Balsas; VII. Los Tuxtlas; VIII. Sierra sur de Michoacán; IX. Sierra norte de Oaxaca; X. Cordillera Costera del sur; XI. Sierra sur de Chiapas; XII. Montaña del norte y altos de Chiapas; XIII. Cañadas de Ocosingo. 
área de cada polígono; como ya se indicó antes, el BMM tiene distribución fragmentada. Adicionalmente se elaboraron mapas de distribución por género y secciones (en el caso de Quercus), mediante el programa ArcView 3.2 (ESRI, 2000), para conocer la distribución general de los taxa señalados.

Para el análisis de similitud florística y de diversidad $\beta$ entre las 13 regiones del BMM propuestas por la CONABIO, se elaboró una matriz con las especies de Fagaceae, y su presencia o ausencia en las regiones prioritarias señaladas. Su presencia se codificó como 1 y su ausencia como 0 . La matriz se analizó mediante el índice de similitud Jaccard, posteriormente se construyó un dendrograma que agrupa las regiones de acuerdo con su similitud, por medio del sistema UPGMA y utilizando el programa NTSYSpc 2.11T (Rohlf, 2004). El índice de diversidad $\beta$ se obtuvo con la fórmula: 1-Jaccard.

Finalmente, se obtuvo información de las categorías de riesgo en las que se encuentran las especies de Fagaceae del $\mathrm{BMM}$ con base en la lista roja de los árboles mexicanos del bosque nublado (González-Espinosa et al., 2011), de la Norma Oficial Mexicana para la Protección de Especies Nativas de México (SEMARNAT, 2010) y de la Unión Internacional para la Conservación de la Naturaleza (IUCN, 2010).

\section{Resultados}

Riqueza florística. En el BMM de México crecen los géneros Fagus y Quercus, el primero está representado por Fagus grandifolia subsp. mexicana, mientras que Quercus tiene 46 especies que crecen en esta comunidad (Apéndice 1), lo que equivale al 28.6\% de las 161 especies de Fagaceae nativas de México (Valencia-A., 2004). De éstas, la mayor proporción se ubica en la sección Lobatae (32 especies), los llamados encinos rojos, y 14 pertenecen a la sección Quercus (encinos blancos; Cuadro 1). Si se consideran las 2,500 especies, que según Rzedowski (1996) crecen en el BMM, como exclusivas o casi exclusivas, la familia Fagaceae contribuye con el $1.9 \%$ de la riqueza de estas especies. Una comparación con el estudio de Luna-Vega y Magallón (2010), en donde citan la presencia de un total de 3,785 especies de plantas, resultaría en que la familia Fagaceae contribuye con un porcentaje del $1.12 \%$ para la flora de este ecosistema. Mientras que la comparación con el bosque húmedo de montaña, para el que Villaseñor (2010) cita la presencia de 6,790 especies, indica que las especies de la familia Fagaceae de este estudio sólo representarían el $0.69 \%$ de este bioma.

Las cifras de dos géneros y 47 especies de Fagaceae del BMM resultan bajas al compararlas con las de las familias más diversas de angiospermas que crecen en este tipo de bosque (Cuadro 2). Sin embargo, si la comparación se realiza sólo entre especies arbóreas que crecen en esta comunidad, Fagaceae ocupa el tercer lugar en riqueza, después de Lauraceae (con 71 especies) y de Rubiaceae (60 especies; González-Espinosa et al., 2011). Es importante señalar que,

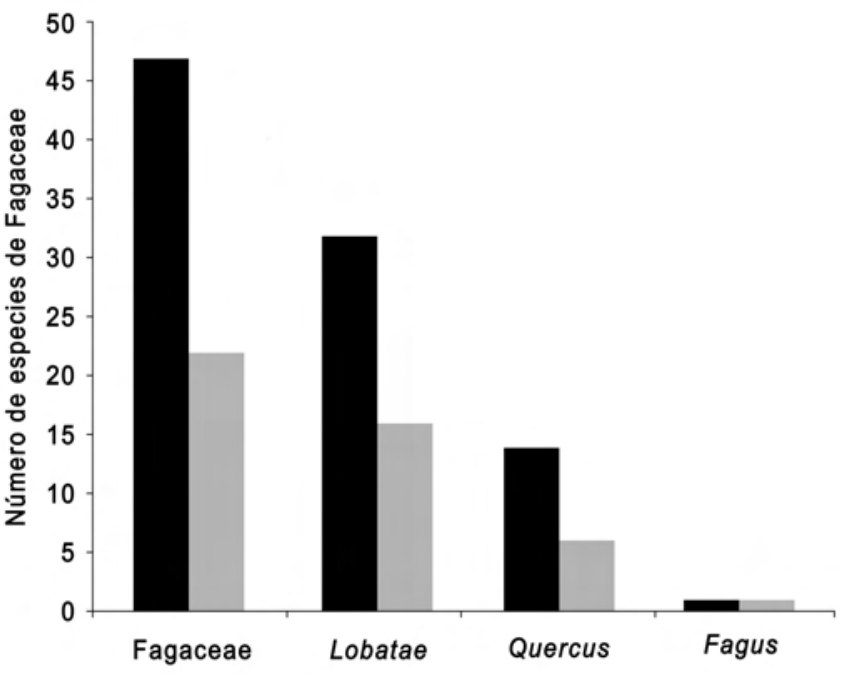

Figura 2. Riqueza de especies de Fagaceae en el BMM y su proporción de especies exclusivas o casi exclusivas. Las barras negras corresponden al total de especies de Fagaceae que crecen en el $\mathrm{BMM}$, las barras grises a las especies que son exclusivas o casi exclusivas.

a diferencia de estas familias que distribuyen su riqueza en ocho y 21 géneros respectivamente, con 46 especies en el BMM, Quercus es el género leñoso con mayor riqueza específica, seguido por Ocotea (Lauraceae) con 26 especies (González-Espinosa et al., 2011).

Distribución general. Además de Fagus grandifolia subsp. mexicana, 22 especies de Quercus (equivalentes al 46.8\% de las especies de Fagaceae que crecen en el BMM) son exclusivas o casi exclusivas de esta comunidad vegetal; de las anteriores, 16 especies pertenecen a la sección Lobatae y sólo seis a la sección Quercus (Figura 2, Cuadro 1).

De las 47 especies de Fagaceae (Apéndice 1), 19 se distribuyen también en Centroamérica (12 de la sección Lobatae y siete de la sección Quercus). Hacia la frontera norte, exceden el límite nacional tres especies: $Q$. rugosa y $Q$. polymorpha de la sección Quercus (que también llegan a Centro América) y Fagus grandifolia; consecuentemente existen 27 especies

Cuadro 1. Especies de Fagaceae del BMM por secciones y género y su distribución.

\begin{tabular}{lcccc}
\hline & $\begin{array}{c}\text { No endémicas, } \\
\text { ni exclusivas }\end{array}$ & $\begin{array}{c}\text { Exclusivas } \\
\text { o casi } \\
\text { exclusivas }\end{array}$ & $\begin{array}{c}\text { Endémicas } \\
\text { de México }\end{array}$ & $\begin{array}{c}\text { Endémicas y } \\
\text { exclusivas o } \\
\text { casi exclusivas }\end{array}$ \\
\hline $\begin{array}{l}\text { Sección } \\
\text { Lobatae }\end{array}$ & 12 & 16 & 20 & 10 \\
$\begin{array}{l}\text { Sección } \\
\text { Quercus }\end{array}$ & 7 & 6 & 7 & 3 \\
Fagus & 0 & 1 & 0 & 0 \\
TOTAL & 19 & 23 & 27 & 13 \\
\hline
\end{tabular}




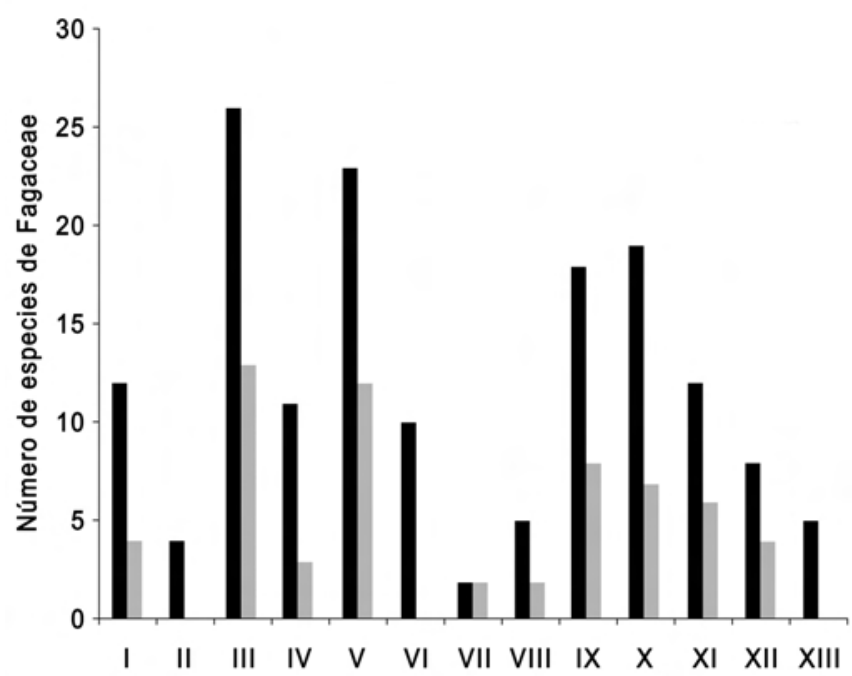

Figura 3. Riqueza de especies de Fagaceae en cada región prioritaria (barras negras) para la conservación del BMM y su proporción de especies exclusivas o casi exclusivas en cada región.

de Fagaceae que sólo se distribuyen en México (Cuadro 1), en donde los encinos de la sección Lobatae son mayoría con 20 especies, diez de ellas exclusivas o casi exclusivas de este ecosistema, mientras que de la sección Quercus, siete especies están restringidas a México, y de ellas, tres son exclusivas o casi exclusivas del BMM (Apéndice 1), lo que equivale al 29.6\% (13 especies) de Fagaceae.

Las Fagaceae que no son exclusivas del BMM, se extienden a regiones templadas y húmedas del bosque de Quercus (17 especies), bosque de coníferas (14 especies) y bosque mixto de coníferas y Quercus (19 especies).

Distribución por regiones. El análisis de la distribución de las especies de Fagaceae en las 13 regiones prioritarias propuestas por la CONABIO (2010), muestra que la Huasteca Alta Hidalguense (región III), presenta la mayor diversidad de especies de la familia, pues alberga 26 de las 47 especies de Fagaceae del BMM, con 13 exclusivas o casi exclusivas de esta comunidad. En segundo lugar está la región del Centro de Veracruz (región V), con 23 especies, 12 exclusivas o casi exclusivas. La Sierra Norte de Oaxaca (región IX) y Cordillera Costera del Sur (región X) tienen una riqueza de 18 y 19 especies respectivamente (Figura 3).

Las regiones con menor riqueza de especies de Fagaceae corresponden a la de Los Tuxtlas (región VII), con dos especies, Quercus skinnerii y $Q$. corrugata, ambas exclusivas y con requerimientos altos de humedad. Cabe señalar que algunos especímenes consultados de estas especies señalan que pueden formar parte del bosque tropical perennifolio. Otra región que también mostró poca riqueza específica de encinos fue la de la Sierra sur de Michoacán (región VIII), con cinco especies: $Q$. candicans, $Q$. elliptica, $Q$. peduncularis, $Q$. martinezii y $Q$. uxoris, las dos últimas exclusivas o casi exclusivas. La tercera zona con baja riqueza corresponde a las Serranías de Nayarit (región II), que sólo incluye a $Q$. candicans, $Q$. castanea, $Q$. elliptica y $Q$. scytophylla, ninguna de ellas exclusiva. Otra región con baja riqueza es la de Las Cañadas de Ocosingo (región XIII), que alberga cinco especies: $Q$. candicans, $Q$. crassifolia, $Q$. crispipilis, $Q$. polymorpha y $Q$. segoviensis, ninguna exclusiva (Figura $3)$. Es necesario resaltar que siete especies de encinos: $Q$. duratifolia, Q. hirtifolia, Q. iltisii, Q. macdougallii, Q. mиllerii, $Q$. nixoniana y $Q$. rubramenta, son endémicas a sólo una de las regiones prioritarias.

Fagus grandifolia subsp. mexicana limita su distribución a los BMM más húmedos de la Sierra Madre Oriental Plegada (región I), la Huasteca Alta Hidalguense (región III) y el Centro de Veracruz (región V; Figura 4). De acuerdo con Denk et al. (2005), el género tuvo mayor extensión durante el Mioceno, lo que aunado a los datos de similitud mostrados más adelante, sugiere que estas tres regiones estuvieron conectadas en el pasado; en la actualidad, la distribución de Fagus en México corresponde a sus límites australes. La limitada concentración de Fagus contrasta con la del género Quercus, que se distribuye en todas las regiones prioritarias del BMM. La comparación de la distribución de los encinos rojos (Figura 5) respecto a la de los blancos (sección Quercus, Figura 6), muestra que a excepción de las regiones II y VIII, en donde no se encontraron registros de la sección Quercus, en el resto, ambas secciones están representadas. Las figuras 5 y 6 muestran que las colectas en la región XIII son escasas. En ellas también se observa que los sitios de la sección Lobatae son superiores en número que los de la sección Quercus, siendo este dato congruente con las preferencias de hábitat de ambas secciones. Evidentemente hay una relación entre la diversidad de cada género y sección, y la amplitud de la distribución que presentan, así como con las preferencias de hábitat de cada uno.

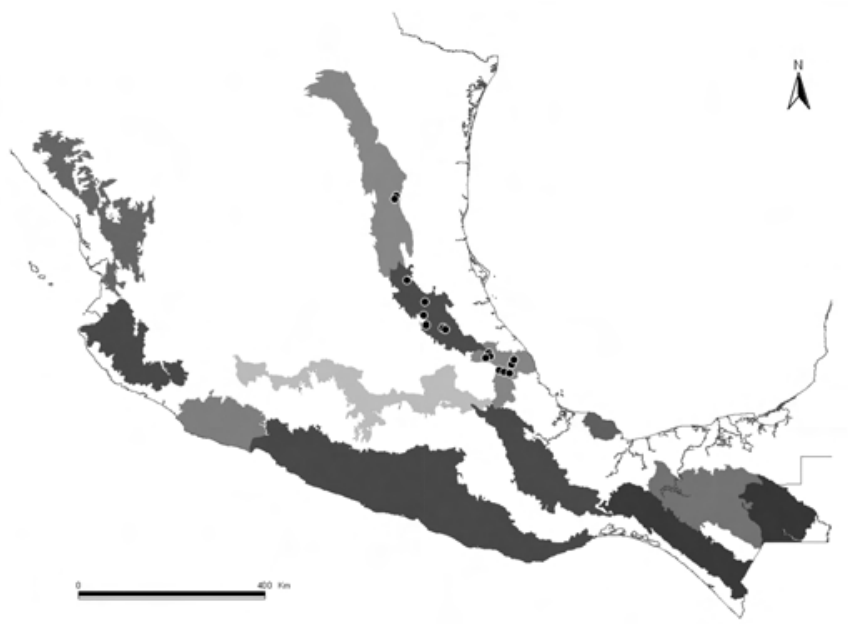

Figura 4. Regiones prioritarias en donde se ha registrado Fagus grandifolia subsp. mexicana en el BMM en México. 




Figura 5. Regiones prioritarias en donde se han registrado especies de la sección Lobatae (encinos rojos) en el BMM en México.

Diversidad $\beta$ y similitud entre las regiones prioritarias. Los índices de similitud obtenidos mediante el índice de Jaccard arrojaron cifras bajas. El índice mayor es el que se presenta entre las regiones contiguas Huasteca Alta Hidalguense (región III) y el Centro de Veracruz (región V), con 0.59. La región VII (Los Tuxtlas) muestra los índices $\beta$ más altos respecto al resto de las regiones, lo que indica un alto recambio de especies entre ellas; los índices $\beta$ entre las 13 regiones van de 1 a 0.41 .

El dendrograma de la Figura 7 muestra cuatro grupos, el grupo I está formado por la Huasteca Alta Hidalguense (región III), el Centro de Veracruz (región V), Sierra Madre Oriental Plegada (I) y la Sierra Norte de Oaxaca (IX). El grupo II lo integran dos subgrupos: IIa, formado por la Serranías de Nayarit (región II) y la Sierra Sur de Michoacán (región VIII); y el grupo IIb, integrado por la Sierra Madre del Sur y la Franja Neovolcánica de Jalisco (región IV), la



Figura 6. Regiones prioritarias en donde se han registrado especies de la sección Quercus (encinos blancos) en el BMM en México.
Cuenca alta del Balsas (región VI) y la Cordillera Costera del Sur (X); en el grupo III están las Montaña del norte y altos de Chiapas (región XII), y las Cañadas de Ocosingo (región XIII) y, el grupo IV lo integran la Sierra de Los Tuxtlas (VII) y la Sierra sur de Chiapas (XI). Este esquema sugiere una relación histórica más reciente entre las regiones de un determinado grupo, con respecto a las de otro grupo del mismo dendrograma.

Especies de Fagaceae en riesgo. La mayoría de las 47 especies de Fagaceae que crecen en el BMM (Apéndice 1) han sido evaluadas, ya sea con los criterios propuestos por la IUCN (2010), o en una posterior evaluación con estos mismos criterios en la Lista Roja de los Árboles mexicanos del Bosque Nublado (González-Espinosa et al., 2011), o en la NOM-059-SEMARNAT-2010 (SEMARNAT, 2010), o incluso, en las tres. Esta última sólo incluye a Fagus grandifolia subsp. mexicana como especie en peligro de extinción y la misma categoría le otorgó la Lista Roja. La IUCN (2010) incluye 30 de las 47 especies de este trabajo, seis de ellas las considera con datos insuficientes (DD); a 13 las califica como especies de preocupación menor (LC), categoría muy cuestionable en el caso de Quercus corrugata, $Q$. cortesii, $Q$. lancifolia y $Q$. trinitatis, que son exclusivas y con poblaciones pequeñas; sólo $Q$. crispipilis es calificada como una especie "en peligro". Por su parte, la más reciente propuesta de González-Espinosa et al. (2011) señala a 38 especies de Fagaceae del BMM con alguna categoría de riesgo; sin embargo, en este estudio sólo se consideran 36 de ellas, ya que dos son descartadas en este trabajo ( $Q$. ocoteifolia y $Q$. vicentensis, como ya se indicó en el método). De acuerdo con el mencionado estudio, nueve especies están en peligro (EN), 12 en peligro crítico (CR), cinco en preocupación menor (LC), dos casi amenazada (NT) y siete en categoría de vulnerables (VU). En el mismo trabajo se señala a $Q$. $m u$ llerii como extinta (EX); sin embargo, en 2010 el equipo de trabajo de la Dra. Cecilia A. Corrado, de la Universidad de la Sierra de Juárez (UNSIJ), en el estado de Oaxaca, colectó esta especie en la localidad tipo y en otra localidad cercana a la anterior y comentó que la población, aunque formada por pocos árboles, está celosamente cuidada por los pobladores que viven cerca (C. A. Corrado, com. pers.).

\section{Discusión}

La falta de un adecuado conocimiento taxonómico de las especies de la familia Fagaceae en México o identificaciones erróneas, condujeron a sobreestimar su diversidad en el BMM. Varios trabajos citan a Quercus acutifolia, Q. deserticota, $Q$. glaucoides y $Q$. magnoliifolia, entre otras, como especies que crecen en esta comunidad; sin embargo, estas especies son características de otros tipos de vegetación con estacionalidad marcada y no forman parte del BMM. La actualización del conocimiento de datos básicos, como 


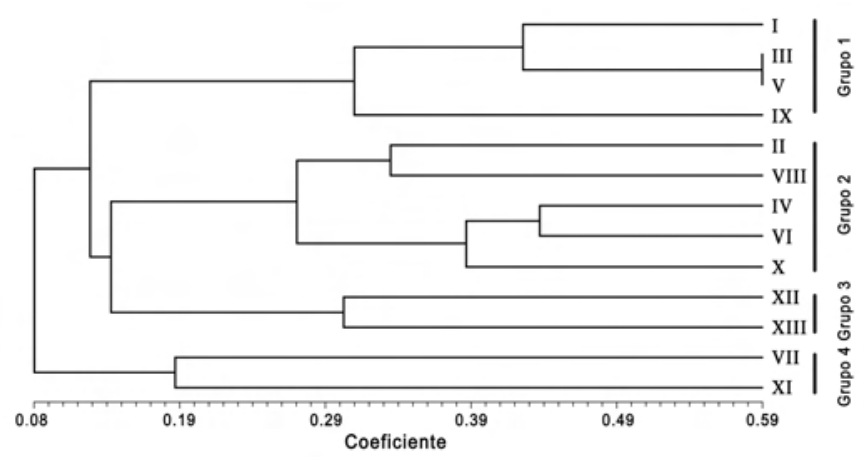

Figura 7. Agrupación, mediante índices de similitud, de las 13 regiones prioritarias para la conservación del BMM con datos de la presencia de Fagaceae.

son los de la riqueza específica de alguna entidad, en este caso la riqueza de la familia Fagaceae en el BMM, permite disponer de información que conducirá a la propuesta de estudios futuros de diversa índole. Este estudio encontró 47 especies de la familia Fagaceae concentradas en el género Quercus, que incluye 46 especies. Éste es el género leñoso más diverso de este bosque y resalta su importancia ecológica en el mismo.

Fagus grandifolia subsp. mexicana es el único taxon del género que se encuentra de forma fragmentada en las regiones prioritarias I, III y V propuestas por la CONABIO (Figura 4), y aunque Téllez-Valdés et al. (2006) mencionan que potencialmente podría llegar hasta Oaxaca, el género no se ha encontrado en dicha entidad. De acuerdo con WilliamsLinera et al. (2003) y Denk et al. (2005), el género llegó a México durante el Mioceno, y hasta América del Sur durante el Plioceno, y ocupa desde entonces, hábitats similares a los actuales. Posteriormente, su distribución disminuyó debido a los cambios climáticos. Las poblaciones mexicanas quedaron aisladas de las de América del Norte hace aproximadamente siete millones de años (Manos y Stanford, 2001). Las poblaciones mexicanas son los relictos más australes que presenta Fagus. Sus requerimientos ambientales específicos y su reducida diversidad pueden ser la causa de la limitada distribución que este género presenta en México y que contrasta con la de Quercus, que alcanza en el país su mayor diversidad y ocupa una gran variedad de ambientes.

En el género Quercus, las especies de la sección Lobatae (encinos rojos), que crecen en el BMM, superan en número a las especies de la sección Quercus (encinos blancos). Este resultado es congruente con lo mencionado por Nixon (1993a), quien señala que los encinos rojos son más diversos en los bosques templados y húmedos que los encinos blancos, que tienen su mayor diversidad en climas estacionales. Cabe señalar que Lobatae es la sección más tempranamente divergente del género Quercus, mientras que la sección Quercus es la última del género en divergir (Nixon, 1993b; Manos et al., 1999). Esta distribución resulta interesante al analizarse a la luz de la composición filogenética de angios- permas en el BMM, ya que se ha sugerido que los taxa basales de angiospermas podrían tener mayor representatividad en dicho bosque (Luna-Vega y Magallón, 2010).

La mayoría de las especies de Fagaceae que crecen en el BMM restringen su distribución a México, lo que sugiere una posible evolución in situ. También destaca la presencia de 18 especies de Quercus que se comparten con Centroamérica, lo anterior puede implicar una relación estrecha entre los BMM mexicanos y centroamericanos, y sugiere una afluencia de especies desde Centroamérica hasta México o viceversa. La decisión del sentido del flujo requiere de estudios filogeográficos adicionales. También será necesario tomar en cuenta los factores que han influido en la distribución de los encinos, tales como los cambios climáticos ocurridos a nivel mundial (Good, 1974) y los posibles tiempos de migración (Sauer, 1988). Estos datos contribuirían a dilucidar la historia de las regiones involucradas, y permitirían conocer si las especies de Fagaceae están ahí porque la región les sirvió como refugio o si son de reciente ingreso.

En este trabajo se muestra a La Huasteca Alta Hidalguense y al Centro de Veracruz, (oriente y centro oriente de México), como las regiones con mayor riqueza de especies de la familia Fagaceae, dato que coincide con los de Nixon (1993a) y Valencia-A. (2004), quienes señalan a la región oriental de México como la más diversa para el género Quercus. Asimismo, el dato es congruente con las categorías de prioridad propuestas por la CONABIO para la conservación del BMM, que señala a estas dos regiones con categoría de "crítica" y "alta" respectivamente. En apoyo a esta propuesta, estas dos regiones albergan a nueve y siete (respectivamente) de las 13 especies que son endémicas a México y exclusivas o casi exclusivas.

Las 13 regiones prioritarias para el BMM propuestas por CONABIO tienen un recambio de moderado a alto, lo que señala que la mayoría de las regiones prioritarias presentan diferencias en la composición de las especies de Fagaceae. Asimismo, el análisis de agrupación de las 13 regiones (Figura 7) permite ver que se forman grupos que incluyen las regiones del este, las del oeste o las del sur, lo que sugiere relaciones históricas o conexiones entre algunas de las re-

Cuadro 2. Familias con mayor riqueza en el BMM. $\left(^{\circ}\right)$ Datos de LunaVega y Magallón, 2010. (*) Datos de González-Espinosa et al., 2011.

\begin{tabular}{lccc}
\hline Familia & $\begin{array}{c}\text { Total de } \\
\text { especies }\end{array}$ & $\begin{array}{c}\text { Géneros } \\
\text { arbóreos }\end{array}$ & $\begin{array}{c}\text { Especies } \\
\text { arbóreas }\end{array}$ \\
\hline Asteraceae & $405^{\circ}$ & $8^{*}$ & $11^{*}$ \\
Orchidaceae & $296^{\circ}$ & $0^{*}$ & $0^{*}$ \\
Fabaceae & $255^{\circ}$ & $12^{*}$ & $35^{*}$ \\
Rubiaceae & $163^{\circ}$ & $21^{*}$ & $60^{*}$ \\
Lauraceae & $43^{\circ}$ & $8^{*}$ & $71^{*}$ \\
Fagaceae (en este trabajo) & 47 & 2 & 46 \\
\hline
\end{tabular}


Cuadro 3. Grado de endemismo de las especies de Fagaceae del BMM.

\begin{tabular}{lcccc}
\hline & $\begin{array}{c}\text { Endémicas de } \\
\text { la vertiente } \\
\text { del Golfo/ } \\
\text { exclusivas } \\
\text { del BMM }\end{array}$ & $\begin{array}{c}\text { Endémicas de } \\
\text { la vertiente } \\
\text { del Pacífico/ } \\
\text { exclusivas } \\
\text { del BMM }\end{array}$ & $\begin{array}{c}\text { Especies } \\
\text { endémicas } \\
\text { de México } \\
\text { presentes } \\
\text { en ambas } \\
\text { vertientes }\end{array}$ & $\begin{array}{c}\text { Especies } \\
\text { presentes } \\
\text { también en } \\
\text { Centroamérica }\end{array}$ \\
\hline $\begin{array}{l}\text { Sección } \\
\text { Lobatae }\end{array}$ & $13 / 7$ & $5 / 3$ & 0 & 12 \\
$\begin{array}{l}\text { Sección } \\
\text { Quercus }\end{array}$ & $4 / 2$ & $1 / 1$ & 2 & 7 \\
Fagus & 0 & 0 & 0 & 0 \\
Totales & $17 / 9$ & $6 / 4$ & 4 & 19 \\
\hline
\end{tabular}

giones y también acentúa una distribución regionalizada de las especies de la familia Fagaceae. Destaca el hecho que de las 13 especies exclusivas o casi exclusivas del BMM y endémicas de México (Cuadro 3), cuatro sólo se distribuyen en el oeste de México: $Q$. martinezii, $Q$. nixoniana, $Q$. rubramenta y $Q$. uxoris, y nueve restringen su presencia a regiones del este del país.

Varias de las especies de Fagaceae del BMM presentan distribución limitada, con pocas poblaciones formadas por pocos individuos, además de que son de crecimiento muy lento y ciclos de vida muy largos (aunque en la mayoría de los casos se desconoce a qué edad alcanzan la madurez reproductiva), y una vez que los árboles alcanzan su etapa de reproducción, sólo producen frutos con una periodicidad que va de cinco a diez años, como en el caso de $Q$. cortesii, $Q$. delgadoana, $Q$. insignis y $Q$. skinerii, que debido al gran tamaño de las bellotas de las dos últimas (alcanzan hasta 4 y $7 \mathrm{~cm}$ de diámetro respectivamente), son recolectadas por los pobladores para hacer artesanías. Otro caso alarmante es el de Fagus grandifolia subsp. mexicana, que sólo produce frutos alrededor de cada siete años; los habitantes de las comunidades rurales cercanas a esta especie están a la expectativa para colectar el fruto y venderlo para consumo humano. La recolecta de bellotas, tanto para la elaboración de artesanías como para el consumo humano, dejan sin semillas a las poblaciones de varias especies para la germinación y posterior reclutamiento, en varios de estos casos las poblaciones en su mayoría están conformadas por árboles maduros, lo que aunado a su distribución restringida y ciclo de vida largo, implicaría atención especial para evitar su extinción.

Aproximadamente el 89\% de las especies de Fagaceae del BMM han sido evaluadas en alguna categoría de riesgo (IUCN, 2010; SEMARNAT, 2010; González-Espinosa et al., 2011); especialmente, 28 (62\%) están consideradas en peligro crítico (CR) o en peligro (EN). Estas cifras dan un panorama alarmante e implican la urgencia de estudios detallados de diversa índole de las especies con énfasis en su biología, eco- logía y distribución específica. Los datos de riqueza, distribución, diversidad, endemismo y exclusividad de las especies de esta familia aquí mostrados permitirán tomar decisiones para priorizar futuros estudios y plantear estrategias particulares para protección y conservación de las mismas.

\section{Conclusiones}

La disponibilidad de bases de datos, la abundante literatura en torno al BMM y acerca de la familia Fagaceae, el acceso a herbarios virtuales y físicos, incluyendo ejemplares tipo, así como la visita a diferentes BMM del país, permitió actualizar el conocimiento taxonómico de la familia Fagaceae en este bosque.

La importancia de la familia se resalta al conocer que son 47 las especies de Fagaceae (más de una tercera parte de las conocidas para México) que crecen en este tipo de vegetación. La distribución de éstas es heterogénea y su recambio entre las 13 regiones prioritarias propuestas por la CONA$\mathrm{BIO}$ es de moderada a alta. Muchas de ellas son exclusivas o casi exclusivas del BMM y endémicas de México y con una distribución limitada a una o pocas regiones prioritarias. Los datos aquí presentados muestran las regiones III y V, de la Sierra Madre Oriental, como las de mayor riqueza de especies de la familia, apoyando así la propuesta de la CONABIO (2010) de prioridad de conservación de los BMM de tales regiones.

Este trabajo también resalta la prioridad que tienen algunas especies de la familia para enfocar en ellas estudios encaminados a conocer su biología, el papel que juegan en el ecosistema y su distribución real y potencial. Un estudio con este último enfoque, para las especies de Fagaceae exclusivas o casi exclusivas y endémicas de México, está siendo desarrollando por la primera autora de este trabajo. Todo ello, sin duda repercutirá en la toma de decisiones en conservación, en el contexto de que las especies de la familia Fagaceae son un grupo clave ecológicamente en el BMM. La respuesta del hombre para enfrentar estas tareas debe acelerarse, ya que su demora puede conducir a daños irreversibles en este tipo de vegetación, ya de por sí amenazado.

\section{Agradecimientos}

A la Comisión Nacional para el Conocimiento y Uso de la Biodiversidad (CONABIO) por proporcionar la base de datos "Sistema de Información de los Bosques Mesófilos de Montaña en México (SI-BMM)" para llevar a cabo este estudio. Por la elaboración de los mapas a Angélica Domínguez Pérez, a Saddan Morales Saldaña por su apoyo en la aplicación de NTSYS, a José Antonio Hernández Gómez por la edición de figuras, a José Luis Villaseñor por la lectura crítica y sus acertadas sugerencias y a dos revisores anónimos cuyos comentarios y sugerencias permitieron mejorar este escrito. 


\section{Literatura citada}

Aguilar R.S.H., Gallardo del A.J.L., Romero G.R., Manríquez R., Gutiérrez L.M., Macías H., Brenner J., Aguirre E. y Arango A. 2001. Línea base de la servidumbre ecológica de Las Cañadas Huatusco, Veracruz. Pronatura. Xalapa.

Alcántara A.O. y Luna V.I. 1997. Florística y análisis biogeográfico del bosque mesófilo de montaña de Tenango de Doria, Hidalgo, México. Anales del Instituto de Biología. Universidad Nacional Autónoma de México. Serie Botánica 68:57-106.

Alcántara A.O. y Luna V.I. 2001. Análisis florístico de dos áreas con bosque mesófilo de montaña en el estado de Hidalgo, México: Eloxochitlán y Tlahuelompa. Acta Botanica Mexicana 54:51-87.

Almeida-Cerino C.M. 2008. Distribución espacial de la comunidad de orquídeas epífitas en la selva alta perennifolia y bosque mesófilo de montaña, en el ejido Villa Guadalupe, Huimangui1lo, Tabasco, México. Tesis licenciatura en Ecología. Universidad de Juárez Autónoma de Tabasco. 99 pp.

Briones V.O.L. 1991. Sobre la flora, vegetación y fitogeografía de la Sierra de San Carlos, Tamaulipas. Acta Botanica Mexicana 16:15-43.

Campos-Villanueva A. y Villaseñor J. L. 1995. Estudio florístico de la porción central del municipio San Jerónimo Coatlán, Distrito de Miahuatlán (Oaxaca). Boletín de la Sociedad Botánica de México 56:95-120.

Cavazos-Camacho C. y Treviño-Garza E.J. 2000. Evaluación de Bosques Mesófilos en el noreste de México. Memorias de la $10^{a}$ Conferencia de Estados Fronterizos México/E.U.A. sobre recreación, áreas protegidas y vida silvestre. Monterrey.

Challenger A. 1998. Utilización y Conservación de los Ecosistemas Terrestres de México, Pasado, Presente y Futuro. Comisión Nacional para el Conocimiento y Uso de la Biodiversidad, Instituto de Ecología, Universidad Nacional Autónoma de México. México, D.F.

Chaverri-Polini A. 1998. Las montañas, la diversidad biológica y su conservación. Unasylva 195:47-54.

CONABIO. Comisión Nacional para el Conocimiento y Uso de la Biodiversidad. 2010. El Bosque Mesófilo de Montaña en México: Amenazas y Oportunidades para su Conservación y Manejo Sostenible. Comisión Nacional para el Conocimiento y Uso de la Biodiversidad, México. D.F.

Constance L. y Breedlove D.E. 1994. Dahliaphyllum, a new arborescent umbellifer from Guerrero. Acta Botanica Mexicana 26:83-87.

Cruz-Cárdenas G., Villaseñor J. L., López-Mata L. y Ortíz E. 2012. Potential distribution of Humid Mountain Forest in Mexico. Botanical Sciences 90:331-340.

Cuevas-Guzmán R. 1994. Flora de la Estación Científica Las Joyas, Municipio de Autlán, Jalisco. México. Tesis de Maestría. Colegio de Posgraduados. Texcoco. 133 pp.

Denk T., Grimm G.W. and Hemleben V. 2005. Patterns of molecular and morphological diferentiation in Fagus (Fagaceae): Phylogenetic implications. American Journal of Botany 92:1006-1016.

Ellison A.M., Bank M.S., Clinton B.D., Colburn E.A., Elliott K., Ford. C.R., Foster D.R., Kloeppel B.D., Knoepp J.D., Lovett G.M., Mohan J., Orwig D.A., Rodenshouse N.L., Sobczak W.V., Stinson K.A., Stone J.K., Swan C.M., Thompson J., Von Holle B. y Webster J.R. 2005. Loss of foundation species: con- sequences for the structure and dynamics of forested ecosystems. Frontiers in Ecology and the Environment 3:479-486.

ESRI. Environmental Scientific Research Institute. 2000. ArcView 3.2 for Windows. Environmental Systems Research Institute. Redlands.

Flores M.G., Jiménez L.J., Madrigal S.X., Moncayo R.F., y Takaki T.F. 1971. Memoria del Mapa de Tipos de Vegetación de la República Mexicana. Secretaria de Recursos Hidráulicos. México, D.F.

Fonseca R.M., Velázquez E. y Domínguez E. 2001. Carrizal de Bravos. Bosque mesófilo de montaña. Estudios Florísticos en Guerrero. No 12. Las Prensas de Ciencias. Facultad de Ciencias. Universidad Nacional Autónoma de México. México, D.F.

González-Espinosa M., Meave J.A., Lorea-Hernández F.G., Ibarra-Manríquez G. y Newton A.C. Eds. 2011. The Red List of Mexican Cloud Forest Trees. Fauna and Flora International. Cambridge.

González-Espinosa M., Meave J.A., Ramírez-Marcial N., ToledoAcevez T., Lorea-Hernández F.G. y Ibarra-Manriquez G. 2012. Los bosques de niebla de México: conservación y restauración de su componente arbóreo. Ecosistemas 21:36-54.

Good R. 1974. The Geography of the Flowering plants. $4^{\mathrm{a}}$ Ed. Longman, Green \& Co. Londres.

IUCN. International Union for Conservation of Nature. 2010. Red List Threatened Species. <http://www.iucnredlist.org> (consultado 8 de diciembre 2010).

Juárez-Medina A.K. 2013. Diversidad alfa y beta de tres bosques mesófilos de monaña de México ubicados en diferentes provincias biogeográficas. Tesis de Maestría. Universidad Nacional Autónoma de México. México D.F. 58 pp.

Johnston M.C., Nixon K., Nesom G.L. y Martínez M. 1989. Listado de plantas vasculares conocidas de la Sierra de Guatemala, Gómez Farías, Tamaulipas, México. Biotam 1:21-33.

Lorea-Hernández F. y Munn-Estrada D.X. 2005. Estudio florístico de los bosques mesófilos de la Sierra Mazateca de Oaxaca, México. Instituto de Ecología, A.C. Comisión Nacional para el Conocimiento y Uso de la Biodiversidad. México, D.F.

Luna I., Almeida L., Villers L.y Lorenzo L. 1988. Reconocimiento florístico y consideraciones fitogeográficas del bosque mesófilo de montaña de Teocelo, Veracruz. Boletín de la Sociedad Botánica de México 48:35-63.

Luna V.I., Alcántara A.O., Espinosa O.D. y Morrone J.J. 1999. Historical relationships of the Mexican cloud forests: a preliminary vicariance model applying Parsimony Analysis of Endemicity to vascular plant taxa. Journal of Biogeography 26:1299-1305.

Luna V.I., Alcántara A.O., Contreras M.R. y Ruiz J.C.A. 2007. Composición y estructura del bosque mesófilo de montaña de Ocuilan, Estado de México-Morelos. En: Luna I., Morrone J.J. y Espinosa D. Eds. Biodiversidad de la Faja Volcánica Transmexicana, pp.173-178, Universidad Nacional Autónoma de México, Comisión Nacional para el Conocimiento y Uso de la Biodiversidad, México, D.F.

Luna-Vega I. y Magallón S. 2010. Phylogenetic composition of Angiosperm diversity in the Cloud Forests of Mexico. Biotropica 42:444-454.

Manos P.S. y Stanford A.M. 2001. The historical biogeography of Fagaceae: Tracking the tertiary history of temperate and subtropical forests of the northern hemisphere. International Journal of Plant Sciences 162:S77-S93.

Manos P.S., Doyle J.J. and Nixon K.C. 1999. Phylogeny, biogeo- 
graphy and processes of molecular differentiation in Quercus subgenus Quercus (Fagaceae). Molecular Phylogenetics and Evolution 12:333-349.

Martínez G.M.J., Cruz D.R. y Ruíz C.A. 2007. Flora vascular de la Sierra de Taxco. En: Luna V.I., Morrone J.J. y Espinosa D. Eds. Biodiversidad de la Faja Volcánica Transmexicana, pp.149171, Universidad Nacional Autónoma de México, Comisión Nacional para el Conocimiento y Uso de la Biodiversidad, México, D.F.

Mayorga R., Luna I. y Alcántara O. 1998. Florística del bosque mesófilo de montaña de Molocotlán, Molango-Xochicoatlán, Hidalgo, México. Boletín de la Sociedad Botánica de México 63:101-119.

Meave J.A., Soto M. A., Calvo L.M., Paz H. y Valencia S. 1992. Análisis sinecológico del bosque mesófilo de montaña de Omiltemi, Guerrero. Boletín de la Sociedad Botánica de México 52:31-77.

Medina G.C., Guevara-Féfer F., Martínez R.M.A., Silva-Sáenz P., Chávez-Carbajal M.A. y García R.I. 2000. Estudio florístico en el área de la comunidad indígena de Nuevo San Juan Parangaricutiro, Michoacán, México. Acta Botanica Mexicana 52:5-41.

Mejía-Domínguez N.R., Meave J.A. y Ruiz-Jiménez C.A. 2004. Análisis estructural de un bosque mesófilo de montaña en el extremo oriental de la Sierra Madre del Sur (Oaxaca), México. Boletín de la Sociedad Botánica de México 73:13-29.

Nixon K.C. 1993a. The genus Quercus in Mexico. En: Ramamoorthy T.P., Bye R., Lot A. y Fa J. Eds. Biological Diversity of Mexico: Origins and Distribution, pp. 447-458, Oxford University Press, Nueva York.

Nixon K.C. 1993b. Infrageneric classification of Quercus (Fagaceae) and typification of sectional names. Annales des Sciences Forestieres 50(suppl):25-34.

SEMARNAT. Secretaría de Medio Ambiente y Recursos Naturales. 2010. NOM-059-SEMARNAT-2010. Protección ambiental-Especies nativas de México de flora y fauna silvestres-Categorías de riesgo y especificaciones para la inclusión, exclusión o cambio-Lista de especies en riesgo.

Olvera-Vargas M. y Figueroa-Rangel B.L. 2012. Estructura diferencial y zonación de bosques montanos dominados por encino en el centro-occidente de México. Ecosistemas 21:74-87.

Orozco-Villa M. 1995. Vegetación del municipio de Temascaltepec, Estado de México. Tesis de Licenciatura. Universidad Nacional Autónoma de México. México. 100 pp.
Ponce-Vargas A., Luna-Vega I., Alcántara-Ayala O. y Ruíz-Jiménez C.A. 2006. Florística del bosque mesófilo de montaña de Monte Grande, Lolotla, Hidalgo, México. Revista Mexicana de Biodiversidad 77:177-190.

Rohlf J.F. 2004. NTSYS-PC 2.11T. Numerical Taxonomy and Multivariate Analysis System. Exeter Software. Nueva York.

Ruíz-Jiménez C.A., Téllez-Valdés O. y Luna-Vega I. 2012. Clasificación de los bosques mesófilos de montaña de México: afinidades de la flora. Revista Mexicana de Biodiversidad 83:11101144.

Rzedowski J. 1978. Vegetación de México. Limusa. México, D.F.

Rzedowski J. 1996. Análisis preliminar de la flora vascular de los bosques mesófilos de montaña de México. Acta Botanica Mexicana 35:25-44.

Sauer J.D. 1988. Plant Migration (the Dynamics of Geographic Patterning in Seed Plant Species). University of California Press. Los Angeles.

Téllez-Valdés O., Dávila-Aranda P. y Lira-Saade R. 2006. The effects of climate change on the long-term conservation of Fagus grandifolia var. mexicana, an important species of the cloud forest in eastern Mexico. Biodiversity and Conservation 15:1095-1107.

Valdez T.V., Foroughbakhch P.R. y Alanís F.G. 2003. Distribución relictual del bosque mesófilo de montaña en el noreste de México. Ciencia UANL 3:360-365.

Valencia A.S., Nixon K.C. y Kelly L.M. 2011. Quercus delgadoa$n a$ (Fagaceae), a new species from the Sierra Madre Oriental, Mexico. Novon: A Journal for Botanical Nomenclature 21:274277.

Valencia-A. S. 2004. Diversidad del género Quercus (Fagaceae) en México. Boletín de la Sociedad Botánica de México 75:33-53.

Vargas-Ajuria Y.A. 1982. Análisis florístico y fitogeográfico de un bosque mesófilo de montaña en Huayacocotla, Ver. Tesis de Licenciatura. Universidad Nacional Autónoma de México. México, D.F. 105 pp.

Villaseñor J.L. 2010. El Bosque Húmedo de Montaña en México y sus Plantas Vasculares: Catálogo Florístico-Taxonómico. Comisión Nacional para el Conocimiento y Uso de la Biodiversidad. Universidad Nacional Autónoma de México. México, D.F.

Williams-Linera G., Rowden A. y Newton A.C. 2003. Distribution and stand characteristics of relict populations of Mexican beech (Fagus grandifolia var. mexicana). Biological Conservation 109:27-36.

Recibido: 17 de marzo de 2013

Aceptado: 20 de agosto de 2013 
Apéndice 1. Lista de especies de Fagaceae del BMM y su categoría de riesgo. El asterisco (*) indica que la especie es exclusiva o casi exclusiva de este tipo de vegetación; el signo de grado $\left(^{\circ}\right)$ indica que es una especie que restringe su distribución a México. Para IUCN, por sus siglas en ingles, DD (datos insuficientes), EN (en peligro), CR (en peligro crítico), VU (vulnerable), NT (casi amenazado), LC (preocupación menos), EX (extinta) y VU (vulnerable). La NOM-059-SEMARNAT-2010 sólo incluye a Fagus grandifolia subsp. mexicana como taxa en peligro de extinción, por lo que no se incluye una columna para ésta.

Taxón

IUCN (2010)

González-Espinosa

et al. (2011)

Fagus grandifolia Ehrh. subsp. mexicana (Martínez) A.E.Murray *

EN

\section{Sección Lobatae}

Q. acherdophylla Trel. *o

CR

Q. affinis Scheidw. ${ }^{\circ}$

VU

Q. benthamii A. DC.*

Q. candicans Née

LC

EN

Q. castanea Née

LC

Q. cortesii Liebm. *

LC

LC

NT

Q. crassifolia Bonpl.

Q. crassipes Bonpl. ${ }^{\circ}$

Q. crispifolia Trel.*

NT

EN

VU

Q. delgadoana Valencia-Ávalos, Nixon \& L.M.Kelly *o

EN

Q. depressa Bonpl.

DD

Q. duratifolia C.H.Müll. ${ }^{\circ}$

DD

Q. elliptica Née

LC

VU

Q. furfuracea Liebm. ${ }^{\circ}$

Q. hirtifolia M.L.Vázquez, Valencia-Ávalos \& Nixon *o

DD

CR

Q. iltisii L.González ${ }^{\circ}$

Q. laurina Bonpl.

LC

Q. mexicana Bonpl. ${ }^{\circ}$

Q. mullerii Martínez ${ }^{\circ}$

Q. nixoniana Valencia-Ávalos \& Lozada-Pérez *o

DD

EX

Q. paxtalensis C.H.Müll.

DD

CR

Q. pinnativenulosa C.H.Müll. *o

NT

CR

Q. rubramenta Trel. *o

Q. rysophylla Weath. *o

Q. sapotifolia Liebm. *

Q. sartorii Liebm. *o

Q. scytophylla Liebm. ${ }^{\circ}$

Q. skinneri Benth.*

Q. trinitatis Trel.*

Q. uxoris McVaugh *o

Q. xalapensis Bonpl. *o

\section{Sección Quercus}

Q. ariifolia Trel. ${ }^{\circ}$

Q. corrugata Hook*

Q. germana Schltdl. *o

Q. glabrescens Liebm. ${ }^{\circ}$

Q. insignis M.Martens \& Galeotti *

Q. laeta Liebm. ${ }^{\circ}$
LC

EN

VU

CR

VU

NT

CR 
Taxón

IUCN (2010)

González-Espinosa

et al. (2011)

Q. lancifolia Cham. \& Schltdl. *

LC

NT

Q. macdougalli Martínez *o

VU

CR

Q. martinezii C.H.Müll. *o

DD

CR

Q. obtusata Bonpl. ${ }^{\circ}$

LC

LC

Q. peduncularis Née

LC

Q. polymorpha Schltdl. \& Cham.

Q. rugosa Née

NT

EN

LC

LC

Q. segovienses Liebm.

LC

VU 